\title{
THE RIEMANN PROBLEM FOR THE SHALLOW WATER EQUATIONS WITH DISCONTINUOUS TOPOGRAPHY*
}

\author{
PHILIPPE G. LEFLOCH ${ }^{\dagger}$ AND MAI DUC THANH TH $^{\ddagger}$
}

\begin{abstract}
We construct the solution of the Riemann problem for the shallow water equations with discontinuous topography. The system under consideration is non-strictly hyperbolic and does not admit a fully conservative form, and we establish the existence of two-parameter wave sets, rather than wave curves. The selection of admissible waves is particularly challenging. Our construction is fully explicit, and leads to formulas that can be implemented numerically for the approximation of the general initial-value problem.
\end{abstract}

Key words. shallow water, conservation law, Riemann problem, discontinuous topography

AMS subject classifications. 35L65, 76N10, 76L05

\section{Introduction}

1.1. Shallow water equations. We consider the one-dimensional shallow water equations

$$
\begin{aligned}
\partial_{t} h+\partial_{x}(h u) & =0, \\
\partial_{t}(h u)+\partial_{x}\left(h\left(u^{2}+g h / 2\right)\right) & =-g h \partial_{x} a, \\
\partial_{t} a & =0,
\end{aligned}
$$

where $h$ denotes the height of the water from the bottom to the surface, $u$ the velocity of the fluid, $g$ the gravity constant, and $a$ the height of the river bottom from a given level. Following LeFloch [18] we supplement the first two balance laws for the fluid with the equation $\partial_{t} a=0$ corresponding to a fixed geometry. Adding the equation $\partial_{t} a=0$ allows us to view the shallow water equations (the first two equations in (1.1)), which form a strictly hyperbolic system of balance laws in nonconservative form, as a non-strictly hyperbolic system of balance laws with a linearly degenerate characteristic field.

We are mainly interested in the case that $a$ is piecewise constant,

$$
a(x)= \begin{cases}a_{L}, & x<0, \\ a_{R}, & x>0,\end{cases}
$$

where $a_{L}, a_{R}$ are two distinct constants. The Riemann problem associated with (1.1) is the initial-value problem corresponding to the initial conditions of

$$
(h, u, a)(x, 0)= \begin{cases}\left(h_{L}, u_{L}, a_{L}\right), & x<0 \\ \left(h_{R}, u_{R}, a_{R}\right), & x>0 .\end{cases}
$$

Since $a$ is discontinuous, the system (1.1) cannot be written in a fully conservative form, and the standard notion of weak solutions for hyperbolic systems of conservation laws does not apply. However, the equations still make sense within the framework introduced in Dal Maso, LeFloch, and Murat [7]. (For a recent review see [19, 20].)

*Received: April 13, 2007; accepted (in revised version): September 7, 2007. Communicated by Shi Jin.

${ }^{\dagger}$ Laboratoire Jacques-Louis Lions \& Centre National de la Recherche Scientifique, Université de Paris 6, 4 Place Jussieu, 75252 Paris, France (LeFloch@ann.jussieu.fr).

$\ddagger$ Department of Mathematics, International University, Quarter 6, Linh Trung Ward, Thu Duc District, Ho Chi Minh City, Vietnam (MDThanh@hcmiu.edu.vn). 
1.2. DLM generalized Rankine-Hugoniot relations. Consider an elementary discontinuity propagating with the speed $\lambda$ and satisfying Equation (1.1). Observe that the Rankine-Hugoniot relation associated with the third equation in (1.1) simply reads

$$
-\lambda[a]=0,
$$

where $[a]:=a_{+}-a_{-}$denotes the jump of the bottom-level function $a$, and $a_{ \pm}$denotes its left- and right-hand traces. Then, we have the following possibilities:

(i) either the component $a$ remains constant across the propagating discontinuity,

(ii) or $a$ changes its levels across the discontinuity and the discontinuity is stationary, i.e., the speed $\lambda$ vanishes.

This observation motivates us to define the admissible elementary waves of the system (1.1). First of all, assume that the bottom level $a$ remains constant across a discontinuity; then, $a$ should be constant in a neighborhood of the discontinuity. Eliminating $a$ from (1.1), we obtain the following system of two conservation laws

$$
\begin{aligned}
\partial_{t} h+\partial_{x}(h u) & =0, \\
\partial_{t}(h u)+\partial_{x}\left(h\left(u^{2}+g h / 2\right)\right) & =0,
\end{aligned}
$$

Thus, the left- and right-hand states are related by the Rankine Hugoniot relations corresponding to (1.4):

$$
\begin{aligned}
-\lambda[h]+[h u] & =0, \\
-\lambda[h u]+\left[h\left(u^{2}+g h / 2\right)\right] & =0,
\end{aligned}
$$

where $[h]:=h_{+}-h_{+}$, etc.

Second, suppose that the component $a$ is discontinuous so that the speed vanishes. Then, the solution is independent of the time variable, and it is natural to search for a solution obtained as the limit of a sequence of time-independent smooth solutions of (1.1). (See below.)

Suppose that $(x, t) \mapsto(h, u, a)$ is a smooth solution of (1.1). Then, the system (1.1) can be written in the following form, as a system of conservation laws for the (now conservative) variables $(h, u, a)$ :

$$
\begin{aligned}
\partial_{t} h+\partial_{x}(h u) & =0, \\
\partial_{t} u+\partial_{x}\left(u^{2} / 2+g(h+a)\right) & =0, \\
\partial_{t} a & =0 .
\end{aligned}
$$

Hence, time-independent solutions of (1.1) satisfy

$$
\begin{aligned}
(h u)^{\prime} & =0, \\
\left(u^{2} / 2+g(h+a)\right)^{\prime} & =0,
\end{aligned}
$$

where the dash denotes differentiation with respect to $x$. Trajectories initiating from a given state $\left(h_{0}, u_{0}, a_{0}\right)$ are given by

$$
\begin{aligned}
h u & =h_{0} u_{0}, \\
\frac{u^{2}}{2}+g(h+a) & =\frac{u_{0}^{2}}{2}+g\left(h_{0}+a_{0}\right) .
\end{aligned}
$$


It follows from (1.8) that the trajectories of (1.7) can be expressed in the form $u=$ $u(h), a=a(h)$. Now, letting $h \rightarrow h_{ \pm}$and setting $u_{ \pm}=u\left(h_{ \pm}\right), a_{ \pm}=a\left(h_{ \pm}\right)$, we see that the states $\left(h_{ \pm}, u_{ \pm}, a_{ \pm}\right)$satisfy the Rankine-Hugoniot relations associated with (1.6), but with zero shock speed:

$$
\begin{array}{r}
{[h u]=0,} \\
{\left[u^{2} / 2+g(h+a)\right]=0,}
\end{array}
$$

The above discussion leads us to define the elementary waves of interest, as follows.

DEFINITION 1.1. The admissible waves for the system (1.1) are the following ones:

(a) the rarefaction waves, which are smooth solutions of (1.1) with constant component a depending only on the self-similarity variable $x / t$;

(b) the shock waves which satisfy (1.5) and Lax shock inequalities and have constant component $a$;

(c) and the stationary waves which have zero speed and satisfy (1.9).

As will be checked later, the system (1.1) is not strictly hyperbolic, as was already observed in the previous work [21]. Recall that therein we studied the Riemann problem in a nozzle with variable cross-section and constructed all the Riemann solutions. The present model is analogous, and our main purpose in the present paper is to demonstrate that the technique in [21] extends to the shallow water model and to construct the solution of the Riemann problem. The lack of strict hyperbolicity and the nonconservative form of the equation make the problem particularly challenging. Some aspects of this problem are also covered by Alcrudo and Benkhaldoun [1]. For works on various related models including scalar conservation laws we refer to $[22,15,14,13,12,9,8,2]$.

1.3. Results and perspectives. As we will show, waves in the same characteristic field may be repeated in a single Riemann solution. This happens when waves cross the boundary of the strictly hyperbolic regions and the order of characteristic speeds changes. We will also show below that the Riemann problem may not always have a solution. The Riemann problem may admit exactly one, or two, or up to three distinct solutions for different ranges of left-hand and right-hand states. Thus, uniqueness does not hold for the Riemann problem, as was already observed for the nozzle flow system.

Each possible construction leads to a solution that depends continuously on the left-hand and right-hand states. This is a direct consequence of the smoothness of the elementary wave curves; by the implicit function theorem, the intermediate waves depend continuously on their left- or right-hand states as well as on the Riemann data. These results agree with [21] which covered fluids in a nozzle with variable cross section.

In the present model, the curve of stationary wave is strictly convex. To find stationary waves, one needs to determine the roots of a nonlinear equation (see the function $\varphi$ in (3.1)) which is convex and, therefore, can be easily computed numerically. The Riemann solver derived in the present paper should be useful in combination with numerical methods for shallow water systems developed in $[3,4,11,10,16,6]$ for which we refer to the lecture notes by Bouchut [5]. 


\section{Background}

2.1. Shallow water equations as a non-strictly hyperbolic system. We now discuss the system (1.1) in the nonconservative variables $U=(h, u, a)$. From (1.6) it follows that, for smooth solutions, (1.1) is equivalent to

$$
\begin{aligned}
& \partial_{t} h+u \partial_{x} h+h \partial_{x} u=0, \\
& \partial_{t} u+g \partial_{x} h+u \partial_{x} u+g \partial_{x} a=0, \\
& \partial_{t} a=0,
\end{aligned}
$$

which can be written in the nonconservative form

$$
\partial_{t} U+A(U) \partial_{x} U=0,
$$

where the Jacobian matrix $A(U)$ is given by

$$
A(U)=\left(\begin{array}{lll}
u & u & 0 \\
g & u & g \\
0 & 0 & 0
\end{array}\right) .
$$

The eigenvalues of $A$ are

$$
\lambda_{1}(U):=u-\sqrt{g h}<\lambda_{2}(U):=u+\sqrt{g h}, \quad \lambda_{3}(U):=0,
$$

and corresponding eigenvectors can be chosen as

$$
\begin{aligned}
& r_{1}(U):=(h,-\sqrt{g h}, 0)^{t}, \quad r_{2}(U):=(h, \sqrt{g h}, 0)^{t}, \\
& r_{3}(U):=\left(g h,-g u, u^{2}-g h\right)^{t} .
\end{aligned}
$$

We see that the first and the third characteristic fields may coincide:

$$
\left(\lambda_{1}(U), r_{1}(U)\right)=\left(\lambda_{3}(U), r_{3}(U)\right)
$$

on a hypersurface in the variables $(h, u, a)$, which can be identified as

$$
\mathcal{C}_{+}:=\{(h, u, a) \mid \quad u=\sqrt{g h}\} .
$$

Similarly, the second and the third characteristic fields may coincide:

$$
\left(\lambda_{2}(U), r_{2}(U)\right)=\left(\lambda_{3}(U), r_{3}(U)\right)
$$

on a hypersurface in the variables $(h, u, a)$, which can be identified as

$$
\mathcal{C}_{-}:=\{(h, u, a) \mid \quad u=-\sqrt{g h}\} .
$$

The third eigenvalue $\left(\lambda_{3}, r_{3}\right)$ is linearly degenerate, and we have

$$
-\nabla \lambda_{1}(U) \cdot r_{1}(U)=\nabla \lambda_{2}(U) \cdot r_{2}(U)=\frac{3}{2} \sqrt{g h} \neq 0, \quad h>0 .
$$

Note also that the first and the second characteristic fields $\left(\lambda_{1}, r_{1}\right),\left(\lambda_{2}, r_{2}\right)$ are genuinely nonlinear in the open half-space $\{(h, u, a) \mid \quad h>0\}$.

It is convenient to set

$$
\mathcal{C}=\mathcal{C}_{+} \cup \mathcal{C}_{-}=\left\{(h, u, a) \mid \quad u^{2}-g h=0\right\},
$$




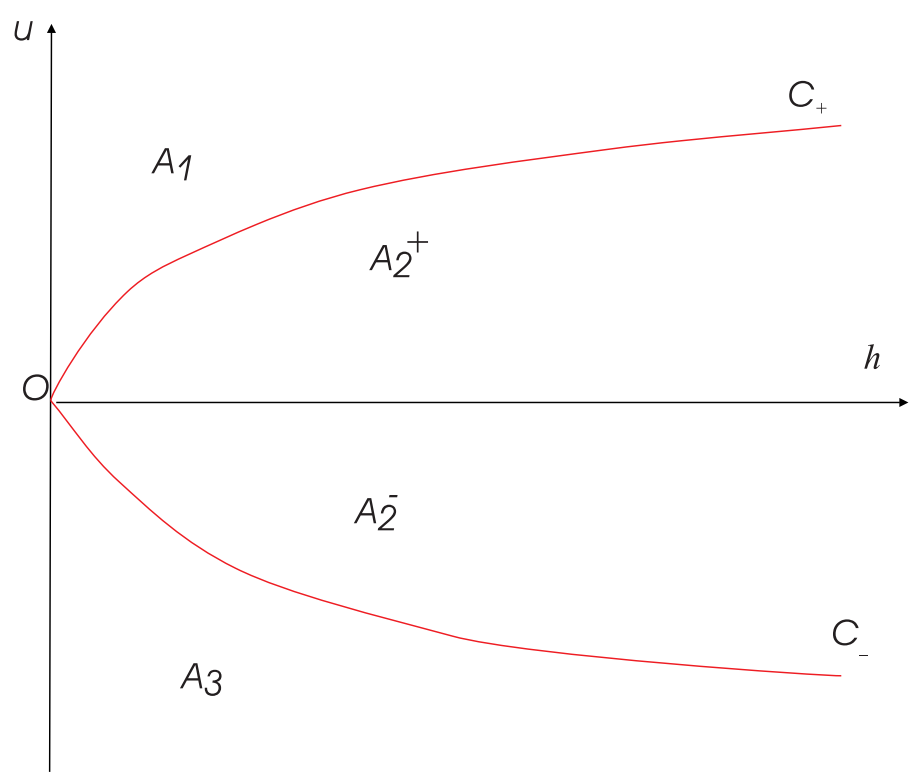

FIG. 2.1. Projection of strictly hyperbolic regions in the $(h, u)$-plane

which is the hypersurface on which the system fails to be strictly hyperbolic.

In conclusion, we have established (cf. Figure 2.1):

Lemma 2.1. On the hypersurface $\mathcal{C}_{+}$in the variables $(h, u, a)$ the first and the third characteristic speeds coincide and, on the hypersurface $\mathcal{C}_{-}$, the second and the third characteristic speeds coincide. Hence, the system (1.1) is non-strictly hyperbolic.

The hypersurface $\mathcal{C}$ divides the phase domain into three disjoint regions, denoted by $A_{1}, A_{2}$ and $A_{3}$, in which the system is strictly hyperbolic. More precisely, we define

$$
\begin{aligned}
& A_{1}:=\left\{(h, u, a) \in \boldsymbol{R}_{+} \times \boldsymbol{R} \times \boldsymbol{R}_{+} \mid \quad \lambda_{2}(U)>\lambda_{1}(U)>\lambda_{3}(U)\right\}, \\
& A_{2}:=\left\{(h, u, a) \in \boldsymbol{R}_{+} \times \boldsymbol{R} \times \boldsymbol{R}_{+} \mid \quad \lambda_{2}(U)>\lambda_{3}(U)>\lambda_{1}(U)\right\}, \\
& A_{2}^{+}:=\left\{(h, u, a) \in A_{2} \mid \quad u>0\right\}, \\
& A_{2}^{-}:=\left\{(h, u, a) \in A_{2} \mid \quad u<0\right\}, \\
& A_{3}:=\left\{(h, u, a) \in \boldsymbol{R}_{+} \times \boldsymbol{R} \times \boldsymbol{R}_{+} \mid \quad \lambda_{3}(U)>\lambda_{2}(U)>\lambda_{1}(U)\right\} .
\end{aligned}
$$

The strict hyperbolicity domain is not connected, which makes the Riemann problem delicate to solve.

2.2. Wave curves. We begin by investigating some properties of the curves of admissible waves.

First, consider shock curves from a given left-hand state $U_{0}=\left(h_{0}, u_{0}, a_{0}\right)$ consisting of all right-hand states $U=(h, u, a)$ that can be connected to $U_{0}$ by a shock wave. Thus, it follows from (1.6) that $U$ and $U_{0}$ are related by the Rankine-Hugoniot relations

$$
\begin{aligned}
-\bar{\lambda}[h]+[h u] & =0, \\
-\bar{\lambda}[h u]+\left[h\left(u^{2}+g h / 2\right)\right] & =0,
\end{aligned}
$$

where $[h]=h-h_{0}$, etc, and $\bar{\lambda}=\bar{\lambda}\left(U_{0}, U\right)$ is the shock speed. 
Fix the state $U_{0}$. A straightforward calculation from the Rankine-Hugoniot relations (2.8) shows that the restriction to the $(h, u)$ plane of the Hugoniot set consists of two curves given by

$$
u=u_{0} \pm \sqrt{\frac{g}{2}}\left(h-h_{0}\right) \sqrt{\left(\frac{1}{h}+\frac{1}{h_{0}}\right)} .
$$

Moreover, along these two curves it holds that

$$
\begin{aligned}
\frac{d u}{d h} & = \pm \sqrt{\frac{g}{2}}\left(\sqrt{\frac{1}{h}+\frac{1}{h_{0}}}-\left(h-h_{0}\right) \frac{1}{2 h^{2} \sqrt{\frac{1}{h}+\frac{1}{h_{0}}}}\right) \\
& \rightarrow \pm \sqrt{\frac{g}{h_{0}}} \text { as } h \rightarrow h_{0} .
\end{aligned}
$$

Since the $i$ th-Hugoniot curve is tangent to $r_{i}\left(U_{0}\right)$ at $U_{0}$, we conclude that the first Hugoniot curve associated with the first characteristic field is

$$
\mathcal{H}_{1}\left(U_{0}\right): \quad u:=u_{1}\left(h, U_{0}\right)=u_{0}-\sqrt{\frac{g}{2}}\left(h-h_{0}\right) \sqrt{\left(\frac{1}{h}+\frac{1}{h_{0}}\right)}, \quad h \geq 0,
$$

while one associated with the second characteristic field is

$$
\mathcal{H}_{2}\left(U_{0}\right): \quad u:=u_{2}\left(h, U_{0}\right)=u_{0}+\sqrt{\frac{g}{2}}\left(h-h_{0}\right) \sqrt{\left(\frac{1}{h}+\frac{1}{h_{0}}\right)}, \quad h \geq 0 .
$$

Along the Hugoniot curves $\mathcal{H}_{1}, \mathcal{H}_{2}$, the corresponding shock speeds are given by

$$
\begin{aligned}
\bar{\lambda}_{1,2}\left(U_{0}, U\right) & =\frac{h u_{1,2}-h_{0} u_{0}}{h-h_{0}} \\
& =u_{0} \mp \sqrt{\frac{g}{2}\left(h+\frac{h^{2}}{h_{0}}\right)}, \quad h \geq 0 .
\end{aligned}
$$

As is customary, the shock speed $\bar{\lambda}_{i}\left(U_{0}, U\right)$ is required to satisfy Lax shock inequalities [17]:

$$
\lambda_{i}(U)<\bar{\lambda}_{i}\left(U_{0}, U\right)<\lambda_{i}\left(U_{0}\right), \quad i=1,2 .
$$

Thus, the 1-shock curve $\mathcal{S}_{1}\left(U_{0}\right)$ initiating from the left-hand state $U_{0}$ and consisting of all right-hand states $U$ that can be connected to $U_{0}$ by a Lax shock associated with the first characteristic field is

$$
\mathcal{S}_{1}\left(U_{0}\right): \quad u=u_{1}\left(h, U_{0}\right)=u_{0}-\sqrt{\frac{g}{2}}\left(h-h_{0}\right) \sqrt{\left(\frac{1}{h}+\frac{1}{h_{0}}\right)}, \quad h>h_{0} .
$$

Similarly, the 2-shock curve $\mathcal{S}_{2}\left(U_{0}\right)$ issuing from a left-hand state $U_{0}$ consisting of all right-hand states $U$ that can be connected to $U_{0}$ by a Lax shock associated with the second characteristic field is

$$
\mathcal{S}_{2}\left(U_{0}\right): \quad u=u_{1}\left(h, U_{0}\right)=u_{0}+\sqrt{\frac{g}{2}}\left(h-h_{0}\right) \sqrt{\left(\frac{1}{h}+\frac{1}{h_{0}}\right)}, \quad h<h_{0} .
$$


We summarize these results in the following proposition.

Lemma 2.2 (Shock wave curves). Given a left-hand state $U_{0}$, the 1-shock curve $\mathcal{S}_{1}\left(U_{0}\right)$ consisting of all right-hand states $U$ that can be connected to $U_{0}$ by a Lax shock is

$$
\mathcal{S}_{1}\left(U_{0}\right): \quad u=u_{1}\left(h, U_{0}\right)=u_{0}-\sqrt{\frac{g}{2}}\left(h-h_{0}\right) \sqrt{\left(\frac{1}{h}+\frac{1}{h_{0}}\right)}, \quad h>h_{0} .
$$

The 2 -shock curve $\mathcal{S}_{2}\left(U_{0}\right)$ consisting of all right-hand states $U$ that can be connected to $U_{0}$ by a Lax shock is

$$
\mathcal{S}_{2}\left(U_{0}\right): \quad u=u_{1}\left(h, U_{0}\right)=u_{0}+\sqrt{\frac{g}{2}}\left(h-h_{0}\right) \sqrt{\left(\frac{1}{h}+\frac{1}{h_{0}}\right)}, \quad h<h_{0} .
$$

In view of the Lax shock inequalities (2.13), we also conclude that the backward 1-shock curve $\mathcal{S}_{1}^{B}\left(U_{0}\right)$ issuing from a right-hand state $U_{0}$ and consisting of all lefthand states $U$ that can be connected to $U_{0}$ by a Lax shock associated with the first characteristic field is

$$
\mathcal{S}_{1}^{B}\left(U_{0}\right): \quad u=u_{1}\left(h, U_{0}\right)=u_{0}-\sqrt{\frac{g}{2}}\left(h-h_{0}\right) \sqrt{\left(\frac{1}{h}+\frac{1}{h_{0}}\right)}, \quad h<h_{0},
$$

Similarly, the backward 2-shock curve $\mathcal{S}_{2}^{B}\left(U_{0}\right)$ issuing from a right-hand state $U_{0}$ and consisting of all left-hand states $U$ that can be connected to $U_{0}$ by a Lax shock associated with the second characteristic field is

$$
\mathcal{S}_{2}^{B}\left(U_{0}\right): \quad u=u_{1}\left(h, U_{0}\right)=u_{0}+\sqrt{\frac{g}{2}}\left(h-h_{0}\right) \sqrt{\left(\frac{1}{h}+\frac{1}{h_{0}}\right)}, \quad h>h_{0},
$$

Next, we discuss the properties of rarefaction waves, i.e., smooth self-similar solutions to the system (1.1) associated with one of the two genuinely nonlinear characteristic fields. These waves satisfy the ordinary differential equation:

$$
\frac{d U}{d \xi}=\frac{r_{i}(U)}{\nabla \lambda_{i} \cdot r_{i}(U)}, \quad \xi=x / t, \quad i=1,2 .
$$

For waves in the first family, we have

$$
\begin{aligned}
& \frac{d h(\xi)}{d \xi}=-\frac{2 h(\xi)}{3 \sqrt{g h(\xi)}}=-\frac{2}{3 \sqrt{g}} \sqrt{h(\xi)}, \\
& \frac{d u(\xi)}{d \xi}=\frac{-2 \sqrt{g h(\xi)}}{-3 \sqrt{g h(\xi)}}=\frac{2}{3}, \\
& \frac{d a(\xi)}{d \xi}=0 .
\end{aligned}
$$

It follows that

$$
\frac{d u}{d h}=-\sqrt{\frac{g}{h}}
$$

therefore, the integral curve passing through a given point $U_{0}=\left(h_{0}, u_{0}, a_{0}\right)$ is given by

$$
u=u_{0}-2 \sqrt{g}\left(\sqrt{h}-\sqrt{h_{0}}\right) .
$$


Moreover, the characteristic speed should increase through a rarefaction fan, i.e.,

$$
\lambda_{1}(U) \geq \lambda_{1}\left(U_{0}\right),
$$

which implies

$$
h \geq h_{0},
$$

Thus, we define a rarefaction curve $\mathcal{R}_{1}\left(U_{0}\right)$ issuing from a given left-hand state $U_{0}$ and consisting of all the right-hand states $U$ that can be connected to $U_{0}$ by a rarefaction wave associated with the first characteristic field as

$$
\mathcal{R}_{1}\left(U_{0}\right): \quad u=v_{1}\left(h, U_{0}\right):=u_{0}-2 \sqrt{g}\left(\sqrt{h}-\sqrt{h_{0}}\right), \quad h \leq h_{0} .
$$

A 1-rarefaction wave is determined by

$$
u=u_{0}+\frac{2}{3}\left(\frac{x}{t}-\frac{x_{0}}{t_{0}}\right)
$$

while $h$ is determined by the equation (2.20) and the component $a$ remains constant.

Similarly, we define the rarefaction curve $\mathcal{R}_{2}\left(U_{0}\right)$ issuing from a given left-hand state $U_{0}$ and consisting of all the right-hand states $U$ that can be connected to $U_{0}$ by a rarefaction wave associated with the second characteristic field as

$$
\mathcal{R}_{2}\left(U_{0}\right): \quad u=v_{2}\left(h, U_{0}\right):=u_{0}+2 \sqrt{g}\left(\sqrt{h}-\sqrt{h_{0}}\right), \quad h \geq h_{0} .
$$

The $u$-component of the 2 -rarefaction wave is determined by $(2.21)$ and the $h$-component is given by $(2.22)$.

We can summarize the above results in:

LEMMA 2.3 (Rarefaction wave curves). Given a left-hand state $U_{0}$, the 1 -rarefaction curve $\mathcal{R}_{1}\left(U_{0}\right)$ consisting of all right-hand states $U$ that can be connected to $U_{0}$ by a rarefaction wave associated with the first characteristic field is

$$
\mathcal{R}_{1}\left(U_{0}\right): \quad u=v_{1}\left(h, U_{0}\right):=u_{0}-2 \sqrt{g}\left(\sqrt{h}-\sqrt{h_{0}}\right), \quad h \leq h_{0} .
$$

The 2 -rarefaction curve $\mathcal{R}_{2}\left(U_{0}\right)$ consisting of all right-hand states $U$ that can be connected to $U_{0}$ by a rarefaction wave associated with the second characteristic field is

$$
\mathcal{R}_{2}\left(U_{0}\right): \quad u=v_{2}\left(h, U_{0}\right):=u_{0}+2 \sqrt{g}\left(\sqrt{h}-\sqrt{h_{0}}\right), \quad h \geq h_{0} .
$$

We will also need backward curves which we define here for completeness. Given a right-hand state $U_{0}$, the 1-rarefaction curve $\mathcal{R}_{1}^{B}\left(U_{0}\right)$ consisting of all left-hand states $U$ that can be connected to $U_{0}$ by a rarefaction wave associated with the first characteristic field is

$$
\mathcal{R}_{1}^{B}\left(U_{0}\right): \quad u=v_{1}\left(h, U_{0}\right):=u_{0}-2 \sqrt{g}\left(\sqrt{h}-\sqrt{h_{0}}\right), \quad h \geq h_{0} .
$$

The 2-rarefaction curve $\mathcal{R}_{2}^{B}\left(U_{0}\right)$ consisting of all left-hand states $U$ that can be connected to $U_{0}$ by a rarefaction wave associated with the second characteristic field is

$$
\mathcal{R}_{2}^{B}\left(U_{0}\right): \quad u=v_{2}\left(h, U_{0}\right):=u_{0}+2 \sqrt{g}\left(\sqrt{h}-\sqrt{h_{0}}\right), \quad h \leq h_{0}
$$


In turn, we are in a position to define the wave curves, as follows:

$$
\begin{aligned}
& \mathcal{W}_{1}\left(U_{0}\right)=\mathcal{S}_{1}\left(U_{0}\right) \cup \mathcal{R}_{1}\left(U_{0}\right), \\
& \mathcal{W}_{1}^{B}\left(U_{0}\right)=\mathcal{S}_{1}^{B}\left(U_{0}\right) \cup \mathcal{R}_{1}^{B}\left(U_{0}\right), \\
& \mathcal{W}_{2}\left(U_{0}\right)=\mathcal{S}_{2}\left(U_{0}\right) \cup \mathcal{R}_{2}\left(U_{0}\right), \\
& \mathcal{W}_{2}^{B}\left(U_{0}\right)=\mathcal{S}_{2}^{B}\left(U_{0}\right) \cup \mathcal{R}_{2}^{B}\left(U_{0}\right) .
\end{aligned}
$$

Some properties of the wave curves are now checked.

Lemma 2.4 (Monotonicity properties). The wave curve $\mathcal{W}_{1}\left(U_{0}\right)$ can be parameterized in the form $h \mapsto u=u(h), h>0$, where the function $u$ is strictly convex and strictly decreasing in $h$. The wave curve $\mathcal{W}_{2}\left(U_{0}\right)$ can be parameterized in the form $h \mapsto u=u(h), h>0$, where the function $u$ is strictly concave and strictly decreasing in $h$.

Proof. We only give the proof for the 1 -wave curve $\mathcal{W}_{1}\left(U_{0}\right)$, the proof for $\mathcal{W}_{2}\left(U_{0}\right)$ being similar. For the shock part $\mathcal{S}_{1}\left(U_{0}\right)$, we have

$$
\frac{d u}{d h}=-\sqrt{\frac{g}{2}} \frac{\frac{1}{2 h}+\frac{1}{h_{0}}+\frac{h_{0}}{2 h^{2}}}{\sqrt{\frac{1}{h}+\frac{1}{h_{0}}}}<0 .
$$

For the rarefaction part $\mathcal{R}_{1}\left(U_{0}\right)$, we have

$$
\frac{d u}{d h}=-\sqrt{\frac{g}{h}}<0 .
$$

This establishes the desired monotonicity property of $\mathcal{W}_{1}\left(U_{0}\right)$.

The convexity of $\mathcal{W}_{1}(U)$ follows from the fact that $d u / d h$ is increasing. Indeed, along the shock part $\mathcal{S}_{1}\left(U_{0}\right)$ it holds that

$$
\frac{d^{2} u}{d h^{2}}=\sqrt{\frac{g}{2}} \frac{\left(\frac{1}{2 h^{2}}+\frac{h_{0}}{h^{3}}\right) \sqrt{\frac{1}{h}+\frac{1}{h_{0}}}+\frac{1}{2 h^{2} \sqrt{\frac{1}{h}+\frac{1}{h_{0}}}}\left(\frac{1}{2 h}+\frac{1}{h_{0}}+\frac{h_{0}}{2 h^{2}}\right)}{\frac{1}{h}+\frac{1}{h_{0}}}>0
$$

and, along the rarefaction part $\mathcal{R}_{1}\left(U_{0}\right)$,

$$
\frac{d^{2} u}{d h^{2}}=\frac{\sqrt{g}}{2 h^{3 / 2}}>0
$$

which completes the proof.

Next, we consider the 3 -curve from a state $U_{0}$, which consists of all states $U$ that can be connected to $U_{0}$ by a stationary wave. As seen in (1.9), $U$ and $U_{0}$ are related by the Rankine-Hugoniot relations

$$
\begin{aligned}
& {[h u]=0} \\
& {\left[u^{2} / 2+g(h+a)\right]=0,}
\end{aligned}
$$

This leads to a natural definition of a curve parameterized in $h$ :

$$
\mathcal{W}_{3}\left(U_{0}\right):\left\{\begin{array}{l}
u=u(h)=\frac{h_{0} u_{0}}{h} \\
a=a(h)=a_{0}+\frac{u^{2}-u_{0}^{2}}{2 g}+h-h_{0} .
\end{array}\right.
$$




\section{Admissibility conditions for stationary waves}

3.1. Two possible stationary jumps. In view of the discussion in the previous section, the states across a stationary wave are constrained by the RankineHugoniot relations (2.26). From a given left-hand state we have to determine the right-hand state, which has three components, determined by the two equations (2.26). Moreover, since the component $a$ changes only through stationary waves (which propagate with zero speed) for given bottom levels $a_{ \pm}$we should solve for $u$ and $h$ in terms of $a$. Thus, we rewrite (2.26) in the form

$$
\begin{aligned}
& u=\frac{h_{0} u_{0}}{h}, \\
& a_{0}-a+\frac{u^{2}-u_{0}^{2}}{2 g}+h-h_{0}=0 .
\end{aligned}
$$

Substituting for $u$ and re-arranging the terms, we obtain

$$
\begin{aligned}
& u=\frac{h_{0} u_{0}}{h}, \\
& a_{0}-a+\frac{u_{0}^{2}}{2 g}\left(\frac{h_{0}^{2}}{h^{2}}-1\right)+h-h_{0}=0 .
\end{aligned}
$$

This leads us to search for roots of the function

$$
\varphi(h):=a_{0}-a+\frac{u_{0}^{2}}{2 g}\left(\frac{h_{0}^{2}}{h^{2}}-1\right)+h-h_{0} .
$$

Let us set

$$
\begin{aligned}
& h_{\min }\left(U_{0}\right):=\left(\frac{u_{0}^{2} h_{0}^{2}}{g}\right)^{1 / 3}, \\
& a_{\min }\left(U_{0}\right):=a_{0}+\frac{u_{0}^{2}}{2 g}\left(\frac{h_{0}^{2}}{h_{\min }^{2}}-1\right)+h_{\min }-h_{0} .
\end{aligned}
$$

Some useful properties of the function $\varphi$ in (3.1) are now derived.

Lemma 3.1. Suppose that $U_{0}=\left(h_{0}, u_{0}, a_{0}\right)$ and a are given with $u_{0} \neq 0$. The function $\varphi:(0,+\infty) \rightarrow \boldsymbol{R}$ is smooth and convex and, for some $h_{\min }$, it is decreasing in the interval $\left(0, h_{\min }\right)$ and is increasing in the interval $\left(h_{\min }, \infty\right)$, with

$$
\lim _{h \rightarrow 0} \varphi(h)=\lim _{h \rightarrow+\infty} \varphi(h)=+\infty .
$$

Furthermore, if $a \geq a_{\min }$ then the function $\varphi$ has two roots $h_{*}\left(U_{0}\right), h^{*}\left(U_{0}\right)$ with $h_{*}\left(U_{0}\right) \leq h_{\min }\left(U_{0}\right) \leq h^{*}\left(U_{0}\right)$. These inequalities are strict whenever $a>a_{\min }\left(U_{0}\right)$.

Proof. The smoothness of the function $\varphi$ and the limiting conditions are obvious. Moreover, we have

$$
\frac{d \varphi(h)}{d h}=-\frac{u_{0}^{2} h_{0}^{2}}{g h^{3}}+1
$$

(for $u_{0} \neq 0$ ), which is positive if and only if

$$
h>\left(\frac{u_{0}^{2} h_{0}^{2}}{g}\right)^{1 / 3}=h_{\min }\left(U_{0}\right) .
$$


This establishes the monotonicity property of $\varphi$. Furthermore, we have

$$
\frac{d^{2} \varphi(h)}{d h^{2}}=\frac{3 u_{0}^{2} h_{0}^{2}}{g h^{4}} \geq 0
$$

which shows the convexity of $\varphi$. If $a>a_{\min }\left(U_{0}\right)$, then $\varphi\left(h_{\min }\left(U_{0}\right)\right)<0$. The other conclusions follow immediately.

It is straightforward to check:

LEMma 3.2. The function $h_{\min }$ satisfies the following inequalities:

$$
\begin{aligned}
& h_{\min }\left(U_{0}\right)>h_{0}, \quad U_{0} \in A_{1} \cup A_{3}, \\
& h_{\min }\left(U_{0}\right)<h_{0}, \quad U_{0} \in A_{2} \text {, } \\
& h_{\text {min }}\left(U_{0}\right)=h_{0}, \quad U_{0} \in \mathcal{C} .
\end{aligned}
$$

The roots $h^{*}$ and $h_{*}$ satisfies the following inequalities:

(i) If $a>a_{0}$, then

$$
h_{*}\left(U_{0}\right)<h_{0}<h^{*}\left(U_{0}\right) .
$$

(ii) If $a<a_{0}$, then

$$
\begin{array}{ll}
h_{0}<h_{*}\left(U_{0}\right) & U_{0} \in A_{1} \cup A_{3}, \\
h_{0}>h^{*}\left(U_{0}\right) & U_{0} \in A_{2} .
\end{array}
$$

The function $a_{\min }\left(U_{0}\right)$ satisfy the following inequalities:

$$
\begin{array}{ll}
a_{\min }\left(U_{0}\right)<a_{0}, & \left(h_{0}, u_{0}\right) \in A_{1} \cup A_{2} \cup A_{3}, \\
a_{\min }\left(U_{0}\right)=a_{0}, & \left(h_{0}, u_{0}\right) \in \mathcal{C}_{ \pm} .
\end{array}
$$

The states that can be connected by stationary waves are characterized as follows.

Proposition 3.3. Fix a left-hand state $U_{0}=\left(h_{0}, u_{0}, a_{0}\right)$ and a right-hand bottom level a.

(i) If $u_{0} \neq 0$ and $a>a_{\min }\left(U_{0}\right)$, then there are two distinct right-hand states,

$$
U_{1,2}:=\left(h_{1,2}\left(U_{0}\right), u_{1,2}\left(U_{0}\right), a\right),
$$

where $u_{i}\left(U_{0}\right):=h_{0} u_{0} / h_{i}\left(U_{0}\right), i=1,2$, that can be connected to $U_{0}$ by a stationary wave satisfying the Rankine-Hugoniot relations.

(ii) If $u_{0} \neq 0$ and $a=a_{\min }\left(U_{0}\right)$, the two states in (i) coincide and we obtain $a$ unique stationary wave.

(iii) If $u_{0} \neq 0$ and $a<a_{\min }\left(U_{0}\right)$, there is no stationary wave from $U_{-}$to a state with level $a$.

(iv) If $u_{0}=0$, there is only one stationary jump defined by

$$
u=u_{0}=0, \quad h=h_{0}+a-a_{0} .
$$


We arrive at an important conclusion on stationary jumps.

Proposition 3.4. For $u_{0} \neq 0$, the state $\left(h_{1}\left(U_{0}\right), u_{1}\left(U_{0}\right)\right)$ belongs to $A_{1}$ if $u_{0}<0$, and belongs to $A_{3}$ if $u_{0}>0$, while the state $\left(h_{2}\left(U_{0}\right), u_{2}\left(U_{0}\right)\right)$ always belongs to $A_{2}$. Moreover, we have

$$
\left(h_{\min }\left(U_{0}\right), u=h_{0} u_{0} / h_{\min }\left(U_{0}\right)\right) \in \begin{cases}\mathcal{C}^{+}, & u_{0}>0 \\ \mathcal{C}^{-}, & u_{0}<0\end{cases}
$$

It is interesting to observe that the shock speed in the genuinely nonlinear characteristic fields will change sign along the shock curves. Therefore, it exchanges its order with the linearly degenerate field, as stated in the following theorem.

Proposition 3.5. (a) If $U_{0} \in A_{1}$, then there exists $\tilde{U}_{0} \in \mathcal{S}_{1}\left(U_{0}\right) \cap A_{2}^{+}$corresponding to $h=\tilde{h}>h_{0}$ such that

$$
\begin{aligned}
& \bar{\lambda}_{1}\left(U_{0}, \tilde{U}_{0}\right)=0, \\
& \bar{\lambda}_{1}\left(U_{0}, U\right)>0, \quad U \in \mathcal{S}_{1}\left(U_{0}\right), h \in\left(h_{0}, \tilde{h}_{0}\right), \\
& \bar{\lambda}_{1}\left(U_{0}, U\right)<0, \quad U \in \mathcal{S}_{1}\left(U_{0}\right), h \in\left(\tilde{h}_{0},+\infty\right) .
\end{aligned}
$$

If $U_{0} \in A_{2} \cup A_{3}$, then

$$
\bar{\lambda}_{1}\left(U_{0}, U\right)<0, \quad U \in \mathcal{S}_{1}\left(U_{0}\right) .
$$

(b) If $U_{0} \in A_{3}$, then there exists $\bar{U}_{0} \in \mathcal{S}_{2}^{B}\left(U_{0}\right) \cap A_{2}^{-}$corresponding to $h=\bar{h}>h_{0}$ such that

$$
\begin{aligned}
& \bar{\lambda}_{2}\left(U_{0}, \bar{U}_{0}\right)=0, \\
& \bar{\lambda}_{2}\left(U_{0}, U\right)>0, \quad U \in \mathcal{S}_{2}^{B}\left(U_{0}\right), h \in\left(h_{0}, \bar{h}_{0}\right), \\
& \bar{\lambda}_{2}\left(U_{0}, U\right)<0, \quad U \in \mathcal{S}_{2}^{B}\left(U_{0}\right), h \in\left(\bar{h}_{0},+\infty\right) .
\end{aligned}
$$

If $U_{0} \in A_{1} \cup A_{2}$, then

$$
\bar{\lambda}_{2}\left(U_{0}, U\right)>0, \quad U \in \mathcal{S}_{2}^{B}\left(U_{0}\right) .
$$

3.2. Two-parameter wave sets. From Proposition 3.4 and the arguments in the previous section, we can now construct wave composites. It turns out that two-parameter wave sets can be constructed. For definiteness, we now illustrate this feature for a particular case. Suppose that $U_{0}=\left(h_{0}, u_{0}, a_{0}\right) \in A_{2}^{+}$. We can take a stationary wave from $U_{0}$ to a state $U_{m}=\left(h_{m}, u_{m}, a_{m}\right) \in A_{2}^{+}$using $h^{*}$, followed by another stationary wave from $U_{m}$ to $U \in A_{1}$ using the corresponding value $h_{*}$; then we continue with 1 -waves, and, as in $A_{1}$, the characteristic speed is positive. Since $a_{m}$ can vary, the set of such states $U$ forms a two-parameter set of composite waves containing first and third waves. Such wave sets were constructed even for strictly hyperbolic systems by Hayes and LeFloch [12].

To make the Riemann problem well-posed, it is necessary to impose an additional admissibility criterion. 
3.3. The monotonicity criterion. Since the Riemann problem for (1.1) may in principle admit up to a one-parameter family of solutions, we now require that the Riemann solutions of interest satisfy a monotonicity condition in the component $a$.

(MC) (Monotonicity Criterion) Along any stationary curve $\mathcal{W}_{3}\left(U_{0}\right)$, the bottom level $a$ is a monotone function in $h$. The total variation of the bottom level component of any Riemann solution must not exceed (and, therefore, is equal to) $\left|a_{L}-a_{R}\right|$, where $a_{L}, a_{R}$ are left-hand and right-hand cross-section levels.

A similar selection criterion was used by Isaacson and Temple $[14,15]$ and by LeFloch and Thanh [21], and by Goatin and LeFloch [8]. Under the transformation (if necessary)

$$
x \rightarrow-x, \quad u \rightarrow-u,
$$

a right-hand state $U=(h, u, a)$ transforms into a left-hand state of the form $U^{\prime}=$ $(h,-u, a)$. Therefore, it is not restrictive to assume that

$$
a_{L}<a_{R}
$$

Lemma 3.6. The Monotonicity Criterion imply that stationary shocks do not cross the boundary of strict hyperbolicity. In other words, we have:

(i) If $U_{0} \in A_{1} \cup A_{3}$, then only the stationary shock based on the value $h_{*}\left(U_{0}\right)$ is admissible.

(ii) If $U_{0} \in A_{2}$, then only the stationary shock using $h^{*}\left(U_{0}\right)$ is admissible.

Proof. Recall that the Rankine-Hugoniot relations associated with the linearly degenerate field (2.27) implies that the component $a$ can be expressed as a function of $h$ :

$$
a=a(h)=a_{0}+\frac{u^{2}-u_{0}^{2}}{2 g}+h-h_{0},
$$

where

$$
u=u(h)=\frac{h_{0} u_{0}}{h} .
$$

Thus, differentiating $a$ with respect to $h$, we find that

$$
\begin{aligned}
a^{\prime}(h) & =\frac{u u^{\prime}(h)}{g}+1=-u \frac{h_{0} u_{0}}{g h^{2}}+1 \\
& =-\frac{u^{2}}{g h}+1,
\end{aligned}
$$

which is positive (resp. negative) if and only if

$$
u^{2}-g h<0 \quad\left(\text { resp. } u^{2}-g h>0\right)
$$

or $(h, u, a) \in A_{2}$ (resp. $\in A_{1}$ or $\left.\in A_{3}\right)$. Thus, in order that $a^{\prime}$ keeps the same sign, the point $(h, u, a)$ must remain on the same side as $\left(h_{0}, u_{0}, a_{0}\right)$ with respect to $\mathcal{C}_{ \pm}$. The conclusions in (i) and (ii) follow. 
It follows from Lemma 3.6 that for a given $U_{0}=\left(h_{0}, u_{0}, a_{0}\right) \in A_{i}, i=1,2,3$, and a level $a$, we can define a unique point $U=(h, u, a)$ so that the two points $U_{0}, U$ can be connected by a stationary wave satisfying the (MC) criterion. We have a mapping

$$
\begin{aligned}
S W(., a):[0, \infty) \times \boldsymbol{R} \times \boldsymbol{R}_{+} & \rightarrow[0, \infty) \times \boldsymbol{R} \times \boldsymbol{R}_{+} \\
U_{0}=\left(h_{0}, u_{0}, a_{0}\right) & \mapsto S W\left(U_{0}, a\right)=U=(h, u, a),
\end{aligned}
$$

such that $U_{0}$ and $U$ can be connected by a stationary wave satisfying the (MC) condition. Observe that this mapping is single-valued except on the hypersurface $\mathcal{C}$, where it is two-valued.

Let us use the following notation: $W_{i}\left(U_{0}, U\right)$ will stand for the $i$ th wave from a left-hand state $U_{0}$ to the right-hand state $U, i=1,2,3$. To represent the fact that the wave $W_{i}\left(U_{1}, U_{2}\right)$ is followed by the wave $W_{j}\left(U_{2}, U_{3}\right)$, we use the notation:

$$
W_{i}\left(U_{1}, U_{2}\right) \oplus W_{j}\left(U_{2}, U_{3}\right) .
$$

\section{The Riemann problem}

In this section we construct the solutions of the Riemann problem, by combining Lax shocks, rarefaction waves, and stationary waves satisfying the admissibility condition (MC).

Recall that for general strictly hyperbolic systems of conservation laws, the solution to the Riemann problem exists when the initial jump is sufficiently small only. That is to say that the right-hand states $U_{R}$ should lie in a small neighborhood of the left-hand state $U_{L}$. However, for the system (1.1), we can cover large data and essentially cover a full domain of existence for any given left-hand state. More precisely, we determine the precise range of right-hand states in which the Riemann solution exists.

4.1. Solutions containing only one wave of each characteristic family.

We begin by constructing solutions containing only one wave corresponding to each characteristic field. This structure of solutions is standard in the theory of strictly hyperbolic system of conservation laws. In the next subsection we will consider solutions that contain up to two waves in the same family. The following theorem deals with the case where the left-hand state $U_{L}$ is in $A_{1}$.

TheOREM 4.1. Let $U_{L} \in A_{1}$ and set $U_{1}:=S W\left(U_{L}, a_{R}\right),\left\{U_{2}\right\}=\mathcal{W}_{1}\left(U_{1}\right) \cap \mathcal{W}_{2}^{B}\left(U_{R}\right)$. Then, the Riemann problem (1.1)-(1.2) admits an admissible solution with the following structure

$$
W_{3}\left(U_{L}, U_{1}\right) \oplus W_{1}\left(U_{1}, U_{2}\right) \oplus W_{2}\left(U_{2}, U_{R}\right),
$$

provided $h_{2} \leq \tilde{h}_{1}$. (Figure 4.1).

Proof. Observe that the set of composite waves $S W\left(\mathcal{W}_{1}\left(U_{L}\right), a_{R}\right)$ consists of three monotone decreasing curves, and each lies entirely in each region $A_{i}, i=1,2,3$. The monotone increasing backward curve $\mathcal{W}_{2}^{B}\left(U_{R}\right)$ therefore may cut the three composite curves at a unique point, or two points, or else does not meet the wave composite set. The Riemann problem therefore may admit a unique solution, two solutions, or no solution.

The state $U_{L}$ belongs to $A_{1}$, and in this region the $\lambda_{3}$ is the smallest of the three characteristic speeds. A stationary wave from $U_{L}=\left(h_{L}, u_{L}, a_{L}\right)$ to $U_{1}=\left(h_{1}, u_{1}, a_{R}\right)$ exists, since $a_{L} \leq a_{R}$. Moreover, by Lemma 3.6, we have $U_{1} \in A_{1}$. 


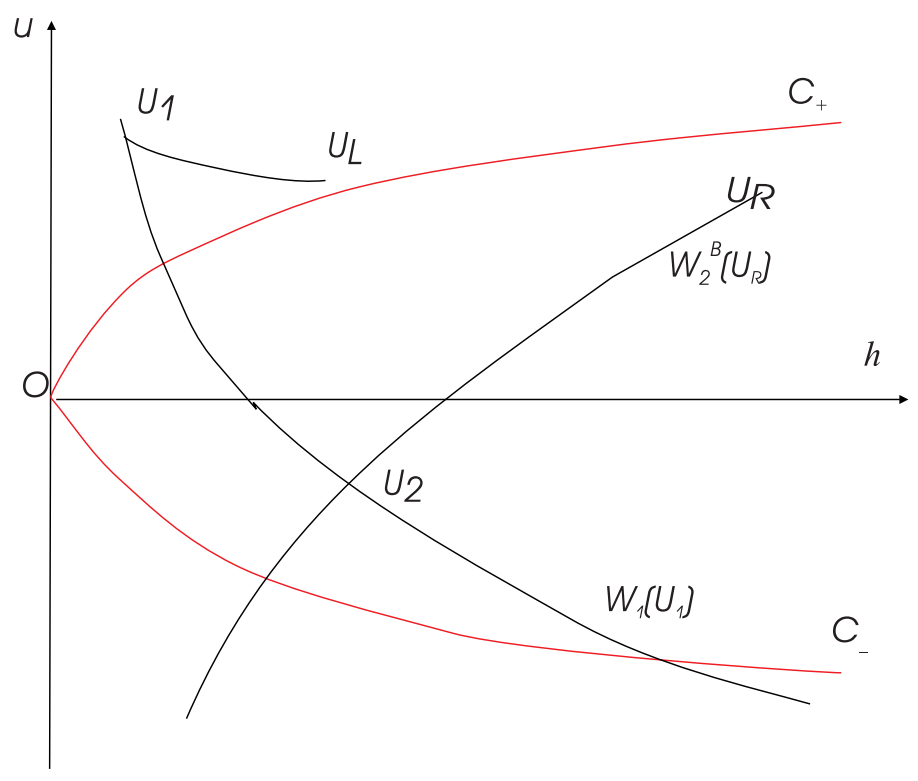

FIG. 4.1. Solution for $U_{L} \in A_{1}$

If $h_{2} \leq h_{1}$, then the stationary wave is followed by a 1-rarefaction wave with positive speed, and then can be continued by a 2 -wave $W_{2}\left(U_{2}, U_{R}\right)$. If $h_{2}>h_{1}$, then the 1-wave in (3.15) is a shock wave. Since $h_{2} \leq \bar{h}_{1}$ and $U_{1} \in A_{1}$, the shock speed $\lambda_{2}\left(U_{1}, U_{2}\right) \geq 0$, and thus it can follow a stationary wave (with zero speed). Moreover, it is derived from (2.12) that

$$
\begin{aligned}
\bar{\lambda}_{1}\left(U_{1}, U_{2}\right) & =u_{1}-\sqrt{\frac{g}{2}\left(h_{2}+\frac{h_{2}^{2}}{h_{1}}\right)} \\
& =\frac{h_{2} u_{2}-h_{1} u_{1}}{h_{2}-h_{1}} \\
& =u_{2}-\sqrt{\frac{g}{2}\left(h_{1}+\frac{h_{1}^{2}}{h_{2}}\right)},
\end{aligned}
$$

thus

$$
\bar{\lambda}_{1}\left(U_{1}, U_{2}\right) \leq u_{2}+\sqrt{\frac{g}{2}\left(h_{R}+\frac{h_{R}^{2}}{h_{2}}\right)}=\bar{\lambda}_{2}\left(U_{2}, U_{R}\right) .
$$

This means that the 1 -shock $S_{1}\left(U_{1}, U_{2}\right)$ can always follow the 2 -shock $S_{2}\left(U_{2}, U_{R}\right)$. A similar condition holds for rarefaction waves. Therefore, the solution structure (3.15) holds.

The following theorem deals with the case where the left-hand state $U_{L}$ is in $A_{1} \cup A_{2}$.

THEOREM 4.2. Let $U_{L} \in A_{1} \cup A_{2}$. Then there exists a region of values $U_{R}$ such that $S W\left(\mathcal{W}_{1}\left(U_{L}\right), a_{R}\right) \cap \mathcal{W}_{2}^{B}\left(U_{R}\right) \neq \emptyset$. In this case this intersection may contain either 


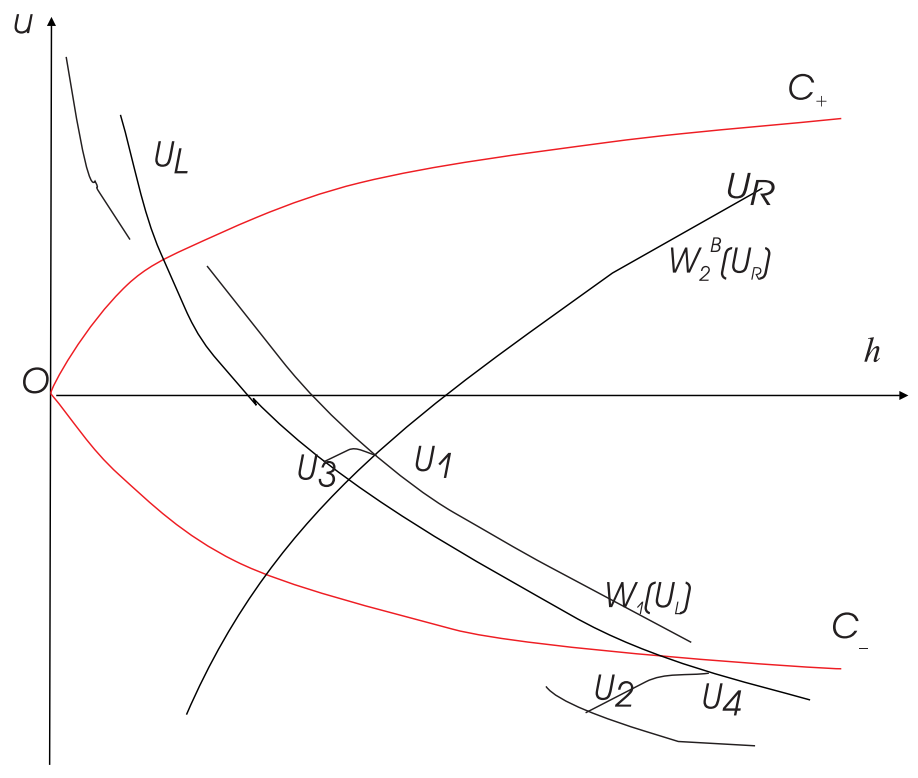

FIG. 4.2. Solution for $U_{L} \in A_{1} \cup A_{2}$

only one or both points $U_{1} \in A_{2}$ and $U_{2} \in A_{3}$. The Riemann problem (1.1)-(1.2) therefore has a solution with the structure

$$
W_{1}\left(U_{L}, U_{3}\right) \oplus W_{3}\left(U_{3}, U_{1}\right) \oplus W_{2}\left(U_{1}, U_{R}\right),
$$

where $U_{3} \in \mathcal{W}_{1}\left(U_{L}\right)$ is the point such that $U_{1}=S W\left(U_{3}, a_{R}\right)$, and also a solution with the structure

$$
W_{1}\left(U_{L}, U_{4}\right) \oplus W_{3}\left(U_{4}, U_{2}\right) \oplus W_{2}\left(U_{2}, U_{R}\right),
$$

where $U_{4} \in \mathcal{W}_{1}\left(U_{L}\right)$ is the point such that $U_{2}=S W\left(U_{4}, a_{R}\right)$, if $h_{2} \geq \bar{h}_{R}$ and $U_{R} \in$ $A_{2}^{-}$. (Figure 4.2)

Proof. The solution may begin with a 1-wave, either a 1-shock with a negative shock speed to a state $U_{3}$, or a 1-rarefaction wave with $\lambda_{1}\left(U_{3}\right) \leq 0$, followed by a stationary wave $W_{3}\left(U_{3}, U_{1}\right)$ from $U_{3}$ to $U_{1}$, then followed by a 2-wave $W_{2}\left(U_{1}, U_{R}\right)$ from $U_{1}$ to $U_{R}$. It is similar in the case of $U_{2}$. However, in order that the stationary wave $W_{3}\left(U_{4}, U_{2}\right)$, for some $U_{4} \in \mathcal{W}_{1}\left(U_{L}\right)$ and $U_{4} \in A_{3}$ obviously, to be followed by a 2-wave $W_{2}\left(U_{2}, U_{R}\right)$, it is required that the wave is a shock with non-negative shock speed $\lambda_{2}\left(U_{2}, U_{R}\right)$. This is equivalent to $h_{2} \geq \bar{h}_{R}$.

THEOREM 4.3. Let $U_{L} \in A_{3}$ and $U_{R} \in A_{1} \cup A_{2}$, and set $U_{1}=S W\left(\mathcal{W}_{2}^{B}\left(U_{R}\right), a_{L}\right) \cap$ $\mathcal{W}_{1}\left(U_{L}\right)$ and $U_{2}=S W\left(U_{1}, a_{R}\right) \in \mathcal{W}_{2}^{B}\left(U_{R}\right)$.

(i) If $U_{1} \in A_{2}^{+} \cup \mathcal{C}_{+} \cup\{u=0\}$, the Riemann problem (1.1)-(1.2) has a solution with the following structure:

$$
W_{1}\left(U_{L}, U_{1}\right) \oplus W_{3}\left(U_{1}, U_{2}\right) \oplus W_{2}\left(U_{2}, U_{R}\right) .
$$




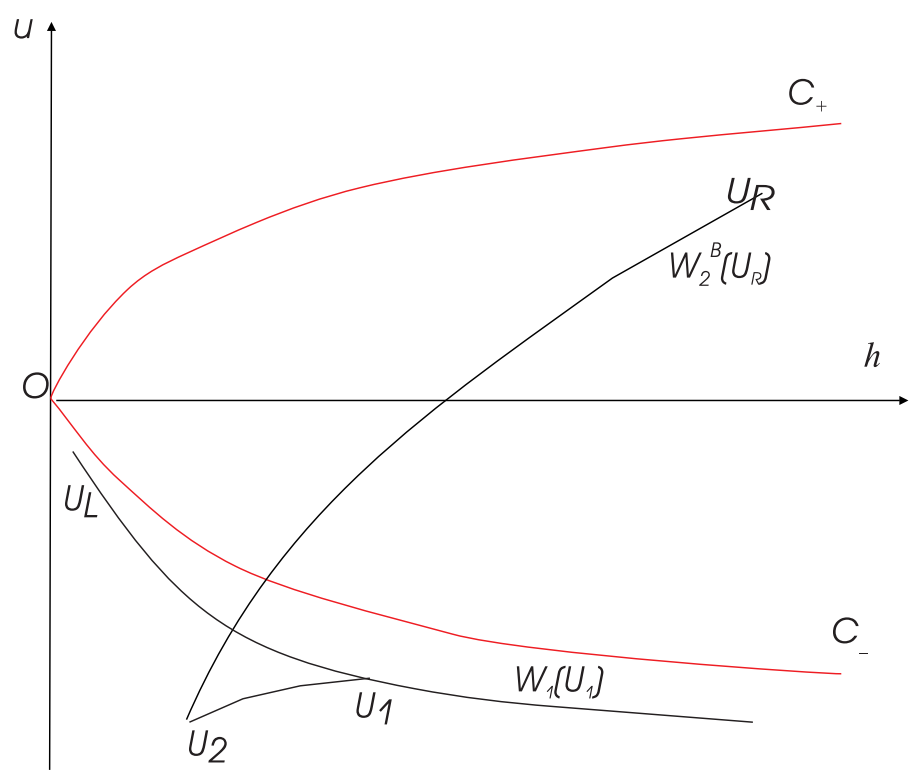

FIG. 4.3. Solution for $U_{L} \in A_{3}$

(ii) If $U_{1} \in A_{2}^{-} \cup \mathcal{C}_{-}$, provided $h_{R} \geq \bar{h}_{2}$, the Riemann solution (4.5) also exists.

(iii) If $U_{1} \in A_{1} \cup A_{3}$, the construction (4.5) does not make sense.

(Figure 4.3).

Proof. If $U_{1} \in A_{2} \cup \mathcal{C}$, the non-positive-speed wave $W_{1}\left(U_{L}, U_{1}\right)$ can be followed by a stationary wave $W_{3}\left(U_{1}, U_{2}\right)$.

When $U_{2} \in A_{2}^{-}$, if $U_{1} \in A_{2}^{+} \cup \mathcal{C}_{+} \cup\{u=0\}$, then this stationary wave can always be followed by a 2 -wave $W_{2}\left(U_{2}, U_{R}\right)$, since the wave speed of the 2-wave is positive. This establishes (i).

If $U_{1} \in A_{2}^{-} \cup \mathcal{C}_{-}$, the wave speed of the 2-wave $W_{2}\left(U_{2}, U_{R}\right)$ is non-negative if and only if $h_{R} \geq \bar{h}_{2}$. This proves (ii).

If $U_{1} \in A_{1}$, the 1-wave has positive speed. So it cannot be followed by a stationary wave. If $U_{1} \in A_{3}$, then $U_{2} \in A_{3}$ by the (MC) criterion. So the 2 -wave $W_{2}\left(U_{2}, U_{R}\right)$ has negative speed. So it cannot be proceeded by a stationary wave. This proves (iii). $\square$

The above theorem enables $U_{R}$ to vary in each region $A_{1}, A_{2}$, and $A_{3}$. The next theorem enables $U_{L}$ to vary in all three regions.

TheOrem 4.4. Let $U_{R} \in A_{3}$. Set $U_{1}=S W\left(U_{R}, a_{L}\right), U_{2}=\mathcal{W}_{2}^{B}\left(U_{1}\right) \cap \mathcal{W}_{1}\left(U_{L}\right)$. A Riemann solution exists and has the following structure:

$$
W_{1}\left(U_{L}, U_{2}\right) \oplus W_{2}\left(U_{2}, U_{1}\right) \oplus W_{3}\left(U_{1}, U_{R}\right),
$$

provided $h_{2} \leq \bar{h}_{1}$. (Figure 4.4).

Proof. The stationary wave $W_{3}\left(U_{1}, U_{R}\right)$ turns out to have the greatest wave speed. In order for this wave to be preceded by the 2 -wave $W_{2}\left(U_{1}, U_{2}\right)$, the wave speed of this 2 -wave has to be non-positive. This is equivalent to the condition $h_{2} \leq \bar{h}_{1}$, according to Proposition (3.5). Similar to (4.4), we have

$$
\lambda_{1}\left(U_{L}, U_{2}\right) \leq \lambda_{2}\left(U_{2}, U_{1}\right),
$$




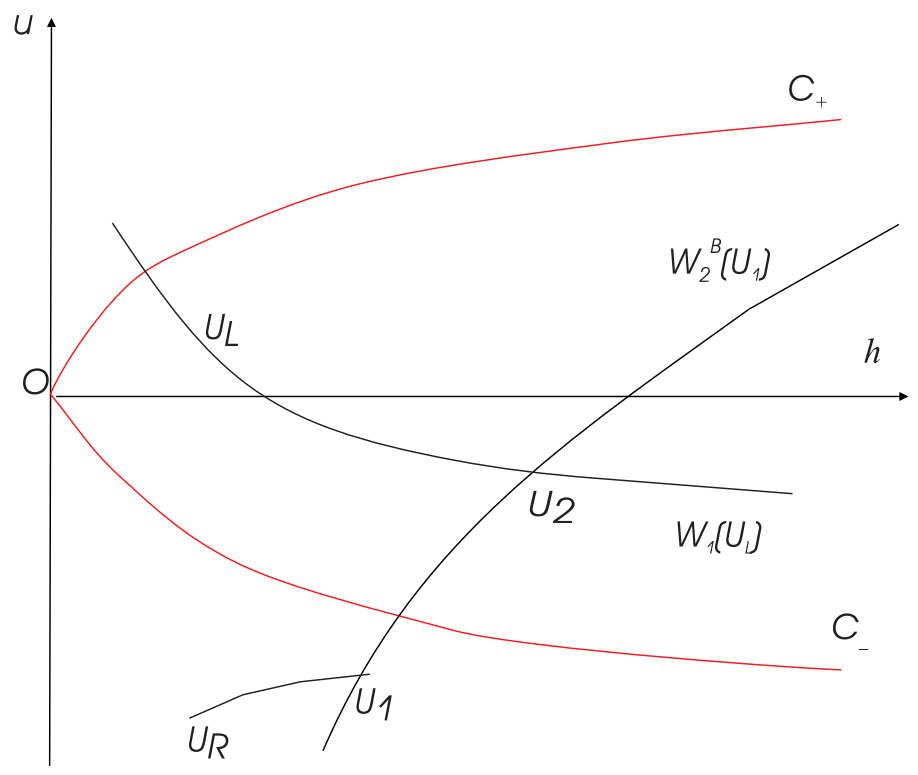

FIG. 4.4. $U_{L}$ may be anywhere

so that the 1 -wave $W_{1}\left(U_{L}, U_{2}\right)$ can follow the 2 -wave $W_{2}\left(U_{2}, U_{1}\right)$.

4.2. Solutions containing more than one wave of each characteristic family. It is remarkable feature of the shallow water system that we can also construct solutions with four elementary waves, using three available characteristic fields. This illustrates one of the difficulties in coping with the Riemann problem when the system under consideration is not strictly hyperbolic.

TheOREM 4.5. Let $U_{L} \in A_{2} \cup A_{3}$ and set $U_{+}=\mathcal{W}_{1}\left(U_{L}\right) \cap \mathcal{C}_{+},\left\{U_{1}\right\}=S W\left(U_{+}, a_{R}\right) \cap$ $A_{1},\left\{U_{2}\right\}=\mathcal{W}_{1}\left(U_{1}\right) \cap \mathcal{W}_{2}^{B}\left(U_{R}\right)$. The Riemann problem (1.1)-(1.2) has a solution with the following structure

$$
R_{1}\left(U_{L}, U_{+}\right) \oplus W_{3}\left(U_{+}, U_{1}\right) \oplus W_{1}\left(U_{1}, U_{2}\right) \oplus W_{2}\left(U_{2}, U_{R}\right),
$$

provided $h_{2} \leq \tilde{h}_{1}$. (Figure 4.5).

THEOREM 4.6. For any $U_{L}$, set $\left\{U_{1}\right\}=S W\left(\mathcal{C}_{-}, a_{R}\right) \cap \mathcal{W}_{2}^{B}\left(U_{R}\right) \cap A_{2}, U_{2}=$ $\left(h_{2}, u_{2}, a_{L}\right) \in \mathcal{C}_{-}$such that $U_{1}=S W\left(U_{2}\right)$, and $\left\{U_{3}\right\}=\mathcal{W}_{2}^{B}\left(U_{2}\right) \cap \mathcal{W}_{1}\left(U_{L}\right)$. Then the Riemann problem (1.1)-(1.2) has a solution with the following structure

$$
W_{1}\left(U_{L}, U_{3}\right) \oplus R_{2}\left(U_{3}, U_{2}\right) \oplus W_{3}\left(U_{2}, U_{1}\right) \oplus W_{2}\left(U_{1}, U_{R}\right),
$$

provided $h_{R} \geq \bar{h}_{1}$ and $h_{3} \leq h_{2}$. (Figure 4.6).

Thus, we see that the Riemann problem (1.1)-(1.2) has a solution consisting of a 1 -wave, a 3 -wave, and two 2 -waves.

It is interesting to note that there are solutions satisfying the (MC) criterion which contain three waves with the same speed (zero). This is the case when a stationary wave jumps from the level $a=a_{L}$ to an intermediate level $a_{m}$ between $a_{L}$ and $a_{R}$, followed by an "intermediate" $k$-shock with zero speed at the level $a_{m}, k=1,3$, and 


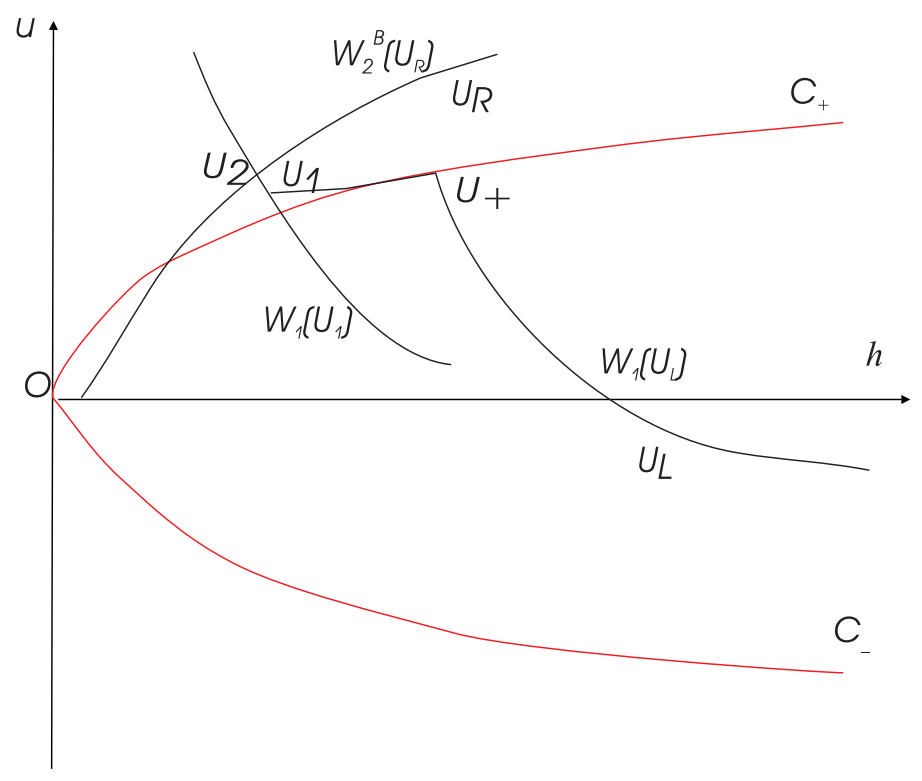

FIG. 4.5. Solution with two 1-waves

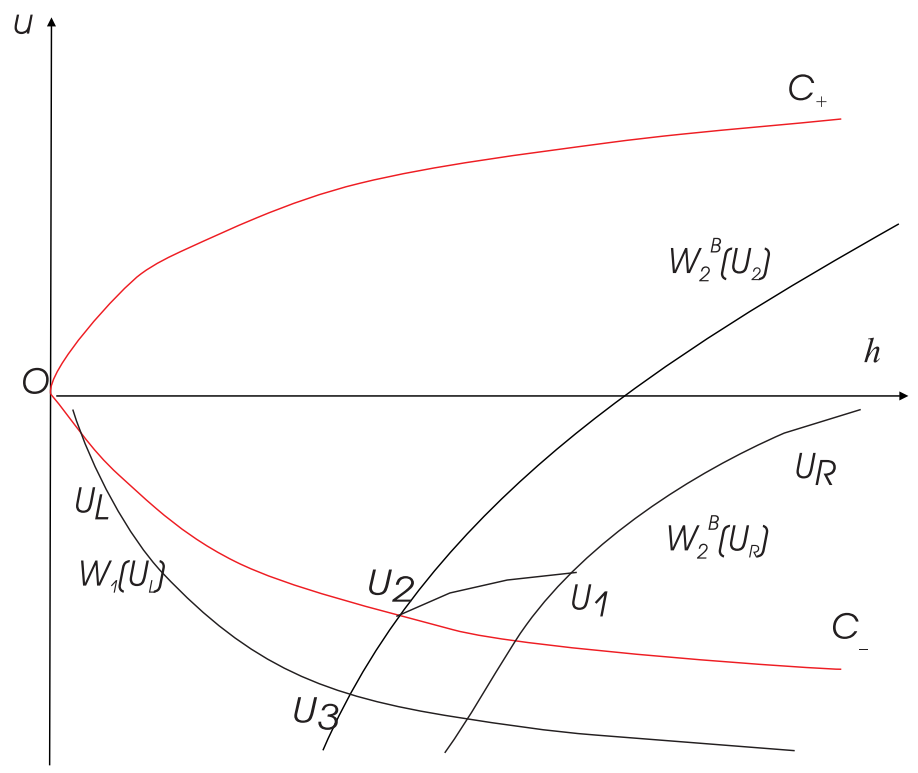

FIG. 4.6. Solution with repeated 2-waves

then followed by another stationary wave jumping from the level $a_{m}$ to $a_{R}$. Thus, there are only two possibilities:

(i) $U_{L}$ belongs to $A_{1}$, and a 1-shock with zero speed is used.

(ii) $U_{R}$ belongs to $A_{3}$, and a 2 -shock with zero speed is used.

We just describe the first case (i), as the second case is similar. Recall from 
Proposition 3.5 that for any $U \in A_{1}$, there exists a unique point denoted by $\tilde{U} \in$ $\mathcal{W}_{1}(U) \cap A_{2}$ such that

$$
\bar{\lambda}_{1}(U, \tilde{U})=0 .
$$

Theorem 4.7. Let $U_{L} \in A_{1}$ and set

$$
\begin{aligned}
& S W\left(U_{L},\left[a_{L}, a_{R}\right]\right):=\cup_{a \in\left[a_{L}, a_{R}\right]} S W\left(U_{L}, a\right), \\
& \widetilde{S W}\left(U_{L},\left[a_{L}, a_{R}\right]\right):=\left\{\tilde{U} \mid U \in S W\left(U_{L},\left[a_{L}, a_{R}\right]\right)\right\} .
\end{aligned}
$$

Whenever

$$
\widetilde{S W}\left(U_{L},\left[a_{L}, a_{R}\right]\right) \cap \mathcal{W}_{2}^{B}\left(U_{R}\right) \neq \emptyset
$$

there exist $a_{m} \in\left[a_{L}, a_{R}\right], U_{1}=S W\left(U_{L}, a_{m}\right)$, and

$$
U_{2} \in \widetilde{S W}\left(U_{L},\left[a_{L}, a_{R}\right]\right) \cap \mathcal{W}_{2}^{B}\left(U_{R}\right)
$$

that define a solution with the structure

$$
W_{3}\left(U_{L}, U_{1}\right) \oplus S_{1}\left(U_{1}, \tilde{U}_{1}\right) \oplus W_{3}\left(\tilde{U}_{1}, U_{2}\right) \oplus W_{2}\left(U_{2}, U_{R}\right) .
$$

Acknowledgments. The first author (P.G.L.) was supported by the A.N.R. Grant 06-2-134423: Mathematical methods in general relativity (MATH-GR) and the Centre National de la Recherche Scientifique (CNRS).

\section{REFERENCES}

[1] A. Alcrudo and F. Benkhaldoun, Exact solutions to the Riemann problem of the shallow water equations with a bottom step, Computers \& Fluids, 30, 643-671, 2001.

[2] D. Amadori, L. Gosse and G. Guerra, Godunov-type approximation for a general resonant balance law with large data, J. Diff. Equ., 198, 233-274, 2004.

[3] N. Andrianov and G. Warnecke, On the solution to the Riemann problem for the compressible duct flow, SIAM J. Appl. Math., 64(3), 878-901, 2004.

[4] E. Audusse, F. Bouchut, M.O. Bristeau, R. Klein and B. Perthame, A fast and stable wellbalanced scheme with hydrostatic reconstruction for shallow water flows, SIAM J. Sci. Comp., 25(6), 2050-2065, 2004.

[5] F. Bouchut, Nonlinear Stability of Finite Volume Methods for Hyperbolic Conservation Laws and Well-Balanced Schemes for Sources, Frontiers in Mathematics, Birkhäuser Verlag, Bäsel, 2004.

[6] M.J. Castro, J.A. García-Rodríguez, J.M. González-Vida and C. Parés, A parallel $2 D$ finite volume scheme for solving the bilayer shallow-water system: modelization of water exchange at the Strait of Gibraltar, Parallel Computational Fluid Dynamics, Elsevier B. V., Amsterdam, 199-206, 2005.

[7] G. Dal Maso, P.G. LeFloch and F. Murat, Definition and weak stability of nonconservative products, J. Math. Pures Appl., 74, 483-548, 1995.

[8] P. Goatin and P.G. LeFloch, The Riemann problem for a class of resonant nonlinear systems of balance laws, Ann. Inst. H. Poincaré Anal. Nonlinéaire, 21, 881-902, 2004.

[9] L. Gosse, Localization effects and measure source terms in numerical schemes for balance laws, Math. Comp., 71, 553-582, 2002.

[10] L. Gosse and G. Toscani, Asymptotic-preserving and well-balanced schemes for radiative transfer and the Rosseland approximation, Numer. Math., 98(2), 223-250, 2004.

[11] J.M. Greenberg, A.Y. Leroux, R. Baraille and A. Noussair, Analysis and approximation of conservation laws with source terms, SIAM J. Numer. Anal., 34, 1980-2007, 1997.

[12] B.T. Hayes and P.G. LeFloch, Nonclassical shock waves and kinetic relations: Strictly hyperbolic systems, SIAM J. Math. Anal., 31, 941-991, 2000. 
[13] T.Y. Hou and P.G. LeFloch, Why nonconservative schemes converge to wrong solutions: error analysis, Math. Comp., 62, 497-530, 1994.

[14] E. Isaacson and B. Temple, Nonlinear resonance in systems of conservation laws, SIAM J. Appl. Math., 52, 1260-1278, 1992.

[15] E. Isaacson and B. Temple, Convergence of the $2 \times 2$ godunov method for a general resonant nonlinear balance law, SIAM J. Appl. Math., 55, 625-640, 1995.

[16] D. Kröner and M.D. Thanh, Numerical solutions to compressible flows in a nozzle with variable cross-section, SIAM J. Numer. Anal., 43(2), 796-824, 2005.

[17] P.D. Lax, Shock waves and entropy, in: E.H. Zarantonello, Contributions to Nonlinear Functional Analysis, 603-634, 1971.

[18] P.G. LeFloch, Shock waves for nonlinear hyperbolic systems in nonconservative form, Institute for Math. and its Appl., Minneapolis, Preprint, 593, 1989.

[19] P.G. LeFloch, Hyperbolic systems of conservation laws: the theory of classical and nonclassical shock waves, Lectures in Mathematics, ETH Zuerich, Birkhauser, 2002.

[20] P.G. LeFloch, Graph solutions of nonlinear hyperbolic systems, J. Hyper. Diff. Equa., 1, 643689,2004

[21] P.G. LeFloch and M.D. Thanh, The Riemann problem for fluid flows in a nozzle with discontinuous cross-section, Commun. Math. Sci., 1(4), 763-797, 2003.

[22] D. Marchesin and P.J. Paes-Leme, A Riemann problem in gas dynamics with bifurcation. Hyperbolic partial differential equations III, Comput. Math. Appl. (Part A), 12, 433-455, 1986. 